\title{
Mean Arterial Pressure
}

National Cancer Institute

\section{Source}

National Cancer Institute. Mean Arterial Pressure. NCI Thesaurus. Code C49679.

The mean pressure of the blood within the arterial circulation. The arterial pressure may be directly measured by insertion of an intra-arterial catheter connected to a transducer. The mean arterial pressure (MAP) can be calculated by subsequent analysis of the waveform. MAP can be approximated without an invasive procedure using the following formula: diastolic pressure plus $1 / 3$ of the pulse pressure, where pulse pressure is systolic pressure - diastolic pressure. 